\title{
O uso da sugestão no diagnóstico e tratamento de cegueira conversiva: um relato de caso
}

The use of suggestion in diagnosis and treatment

of conversion blindness: a case report

Marcus Vinicius Pinto ${ }^{1}$ Adriana Fiszman², Mauricio de Assis Tostes², Marco Antonio Alves Brasil²

\section{RESUMO}

O transtorno conversivo é definido pelo Manual Diagnóstico e Estatístico de Transtornos Mentais - Quarta Edição (DSM-IV) por sintomas que afetam a função sensorial ou motora voluntária, assemelhando-se a uma condição neurológica, mas que, após investigação, não podem ser explicados por essa condição, e sim por fatores psicológicos. Em raras ocasiões, o exame neurológico e os exames complementares não são suficientes para estabelecer o

\section{Palavras-chave}

Transtorno conversivo, diagnóstico, terapêutica, sugestão.

\section{Keywords}

Conversion disorder, diagnosis, therapeutics, suggestion. diagnóstico do transtorno conversivo. Este artigo descreve um caso para o qual a aplicação de uma técnica de sugestão (torpedeamento) confirmou o diagnóstico de cegueira conversiva e também se mostrou eficaz no tratamento. A sugestão, aliada a psicoterapia e psicofarmacologia, pode ser uma ferramenta valiosa no tratamento desses pacientes.

\begin{abstract}
Conversion disorder is defined by Diagnostic and Statistical Manual of Mental Disorders Fourth Edition (DSM-IV) as symptoms affecting voluntary motor or sensory function, resembling a neurological condition, and, after investigation, cannot be explained by this condition, but by psychological factors. On rare occasions, neurological examination and laboratory tests are not sufficient to establish the diagnosis of conversion disorder. This article describes a case for which the use of a technique of suggestion (torpedoing) confirmed the diagnosis of conversion blindness and also proved to be effective for its treatment. Suggestion, combined with psychotherapy and psychopharmacology, can be a valuable tool in the treatment of these patients.
\end{abstract}

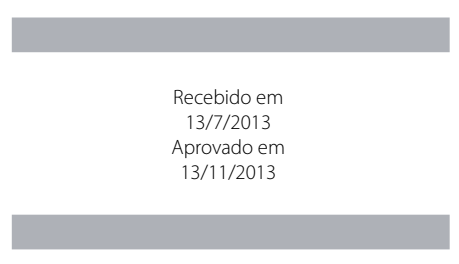

1 Universidade Federal do Rio de Janeiro, Hospital Universitário Clementino Fraga Filho (UFRJ/HUCFF), Serviço de Neurologia. 2 UFRJ/HUCFF, Serviço de Psiquiatria e Psicologia Médica.

Endereço para correspondência: Adriana Fiszman Av. das Américas, 3333, sala 1018, Barra da Tijuca 22631-003 - Rio de Janeiro, RJ, Brasil

E-mail: adriana.fisz@gmail.com 


\section{INTRODUÇÃO}

O transtorno conversivo é definido pelo Manual Diagnóstico e Estatístico de Transtornos Mentais - Quarta Edição (DSM-IV) por sintomas que afetam a função sensorial ou motora voluntária, assemelhando-se a uma condição neurológica, mas que, após investigação, não podem ser explicados por essa condição, e sim por fatores psicológicos’. O diagnóstico é feito por meio do exame neurológico, que inclui testes que revelam inconsistências e incongruências em relação às doenças neurológicas conhecidas. Tais incongruências são suficientes para diagnosticar a maioria dos casos, sem a necessidade de exames complementares. Os transtornos conversivos habitualmente presentes em centros de neurologia são crises não epilépticas psicogênicas, chamadas no nosso meio de pseudocrises ${ }^{2}$, transtornos psicogênicos do movimento ${ }^{3}$, perdas sensoriais de qualquer modalidade, perda da força, alterações da fala ou deglutição, transtornos do equilíbrio e condições neuro-oftalmológicas ${ }^{4}$.

Em raras ocasiões, o exame neurológico e os exames complementares não são suficientes para estabelecer o diagnóstico. Este artigo descreve um caso para o qual a aplicação de uma técnica de sugestão (torpedeamento) selou o diagnóstico de cegueira conversiva e também se mostrou eficaz no tratamento. A avaliação psiquiátrica também é apresentada.

\section{CASO CLÍNICO}

Uma mulher de 56 anos apresentou borramento visual bilateral súbito, com diminuição progressiva da acuidade visual em ambos os olhos, até que, dois meses após, enxergava somente vultos. A avaliação pela oftalmologia descartou doenças oculares. O exame neurológico revelou diminuição acentuada da acuidade visual bilateralmente, apraxia ocular e ataxia óptica. O restante do exame foi normal. Assim, foi feito o diagnóstico de síndrome de Balint, por provável lesão parieto-occipital bilateral. A ressonância magnética de crânio e a análise do líquido cefalorraquidiano foram normais. A paciente foi reexaminada, quando se pôde notar que ela não conseguia pegar a sua própria mão de olhos fechados. Sendo esse um achado incongruente para uma paciente com propriocepção normal, foi levantada a hipótese de cegueira conversiva. Os testes específicos para detectar cegueira conversiva não permitiram a exclusão de uma causa neurológica. O piscar quando o examinador ameaçou a paciente com a própria mão (reflexo de ameaça) e o acompanhar o espelho com os olhos (teste do espelho) seriam esperados em uma paciente com cegueira conversiva (ver referência 5). No entanto, a paciente não piscou e não acompanhou o espelho.

Diante da incerteza do diagnóstico, optou-se por uma técnica de sugestão chamada de torpedeamento. Um dos autores (M.V.P.) informou à paciente que ela tinha dois pontos no cérebro que emitiam "ondas eletromorfofuncionais" e que a impediam de enxergar e movimentar seus olhos. A paciente recebeu a sugestão de que, após a aplicação do diapasão em vibração sobre esses pontos por cinco vezes, essas ondas seriam dissipadas e ela ficaria curada. O procedimento foi realizado em duas ocasiões, e a paciente voltou a movimentar os olhos e a apresentar acuidade visual normal.

Alguns dias após voltar a enxergar, a paciente foi avaliada por uma psiquiatra (A.F.). Queixou-se de tristeza profunda, falta de esperança, excesso de sono, cansaço e isolamento, além de perda de peso e abandono dos cuidados pessoais. Relatou inúmeros traumas emocionais: foi estuprada aos 11 anos pelo irmão de 18 anos, testemunhou a morte de sua mãe aos 13 e aos 14 engravidou de um vizinho de 29 anos. Aos 39 anos, a paciente soube ser portadora do vírus HIV, o que ela vivenciou como sendo o pior evento traumático da sua vida. Nessa época, ela mantinha um relacionamento com um homem que Ihe era infiel e, ao revelar a seu companheiro que ele a havia infectado com o vírus HIV, este reagiu com frieza e a abandonou. Alguns dias antes da perda visual a paciente encontrou-se casualmente com o ex-companheiro, a quem já não via há mais de 10 anos. Ao reconhecê-lo, sentiu "dor, angústia e vontade de sair dali". A paciente expôs seus sintomas e traumas sem variação no tom afetivo da fala e na mímica facial, deixando evidente sua indiferença emocional contrastando com o conteúdo dramático do seu relato. Ao descrever o mal-estar ao se deparar com seu ex-companheiro, sua expressão facial modificou-se, demonstrando tristeza e indignação. Ao final da entrevista, ela confessou o desejo de encontrá-lo para lhe dizer "o mal que ele havia Ihe causado".

Foram formulados os diagnósticos pelo DSM-IV de Transtorno Conversivo com déficit sensorial (cegueira) e Episódio Depressivo Maior grave, sem sintomas psicóticos. A paciente iniciou psicoterapia de base analítica e cloridrato de fluoxetina até $40 \mathrm{mg}$ ao dia. Apresentou remissão do episódio depressivo e não voltou a apresentar perda visual, permanecendo assintomática um ano e meio após a primeira avaliação.

\section{DISCUSSÃO}

Este artigo descreve um caso de transtorno conversivo com déficit sensorial (cegueira) de difícil diagnóstico, assim como sua avaliação psiquiátrica. Os achados anormais do exame neurológico sugeriram uma síndrome neuro-oftalmológica. Outros testes para identificar cegueira conversiva ${ }^{5}$ também foram sugestivos de cegueira neurológica. Os únicos dados que apontaram para cegueira conversiva foram os exames complementares normais e a impossibilidade de alcançar os membros de olhos fechados, um achado incongruente com os testes que mostravam propriocepção normal. 
A confirmação do diagnóstico de transtorno conversivo foi possível pela técnica de sugestão chamada de torpedeamento, que fez com que a paciente recuperasse totalmente a acuidade visual e voltasse a movimentar os olhos. O nome torpedeamento (torpillage) tem origem numa forma persuasiva de terapia criada por Clovis Vincent em 1916 que utilizava correntes elétricas galvânicas e farádicas para fazer com que soldados com "neurose de guerra" retornassem rapidamente para o campo de batalha ${ }^{6}$. Atualmente, o torpedeamento é feito pela aplicação de um estímulo indolor em pontos do corpo onde se apresenta a conversão associada à sugestão ao paciente de que esse estímulo irá curá-lo.

São frequentes os questionamentos sobre a ética dos métodos de sugestão em medicina. Costuma-se alegar que, ao sugestionar o seu paciente, o médico o está "enganando" e tirando-lhe a autonomia. Por outro lado, a sugestão tem um papel crucial no diagnóstico dos transtornos conversivos, já que os neurologistas são unânimes em afirmar que a remissão de um sintoma por sugestão é o sinal mais fidedigno de que aquele sintoma é de natureza conversiva. Por exemplo, as diretrizes diagnósticas para transtorno psicogênico do movimento exigem que o diagnóstico de certeza só seja feito se "o movimento for persistentemente aliviado por psicoterapia, sugestão psicológica ou placebo"' ${ }^{\prime \prime}$. O torpedeamento não somente selou o diagnóstico de cegueira conversiva neste caso como também levou à remissão definitiva do sintoma. Evidências recentes de ensaios randomizados e metanálises mostraram a eficácia da sugestão no tratamento dos fenômenos conversivos ${ }^{8,9}$.

Os métodos de sugestão podem-se tornar valiosas ferramentas terapêuticas nas mãos do psiquiatra, principalmente porque, diferente do que ocorre com a maioria dos transtornos psiquiátricos, não há tratamento farmacológico específico para a conversão. É comum que, ao receber do neurologista um paciente com transtorno conversivo, o psiquiatra indique a psicoterapia sem acompanhar o paciente concomitantemente, acreditando não poder oferecer intervenções que promovam o alívio rápido dos sintomas. No entanto, sendo a conversão um fenômeno crônico e incapacitante na grande maioria dos casos, a possibilidade de lançar mão de uma técnica de sugestão para prontamente aliviar o sofrimento pode modificar o curso da doença, melhorando significativamente o prognóstico e a qualidade de vida, como ocorreu com o caso aqui apresentado.

A entrevista psiquiátrica desse caso revelou que, além de cegueira conversiva, a paciente apresentava episódio depressivo maior. É muito frequente o paciente com transtorno conversivo exibir outro diagnóstico psiquiátrico associado, principalmente os transtornos depressivos e de ansiedade ${ }^{10,11}$. Por exemplo, pacientes com pseudocrises apresentaram taxas elevadas do diagnóstico de transtorno de estresse pós-traumático ${ }^{12,13}$. Portanto, para um grupo de pacientes, mesmo não havendo psicofármacos específicos para conversão, o diagnóstico e o tratamento farmacológico do transtorno psiquiátrico associado podem auxiliar a remissão do sintoma conversivo e também prevenir sua recidiva.

A paciente relatou que a cegueira instalou-se após um encontro casual com o ex-companheiro, a quem a paciente não via há mais de 10 anos. Este a havia infectado com o vírus HIV - pior trauma de sua vida - e a abandonado ao saber que ela portava o vírus. O encontro provocou "dor intensa, angústia e vontade de sair dali" e, ao mesmo tempo, desejo de se aproximar dele. Acredita-se que o fenômeno conversivo seja gerado por conflitos inconscientes que se "convertem" num sintoma neurológico que simboliza o próprio conflito ${ }^{14}$. Nesse caso, é possível que a reação de cegueira diante da visão do ex-companheiro tenha "protegido" a paciente de "enxergar" os sentimentos ambíguos de raiva e amor revividos nesse momento.

Chamou a atenção a indiferença emocional com que a paciente relatou seus sintomas e sua história traumática, chamada classicamente de la belle indifference ${ }^{15}$. Essa indiferença deu lugar à tristeza quando ela falou do sofrimento provocado pelas atitudes do seu ex-companheiro, reforçando, assim, a hipótese de que o sintoma conversivo tenha de fato sido desencadeado pela revivescência das emoções associadas a esse trauma.

\section{CONCLUSÃO}

Este trabalho propôs o uso da sugestão para casos de difícil diagnóstico de transtorno conversivo. O torpedeamento confirmou o diagnóstico de cegueira conversiva e também se mostrou eficaz na sua remissão. A sugestão, aliada a psicoterapia e psicofarmacologia, pode ser uma ferramenta valiosa no tratamento dos pacientes com transtorno conversivo.

\section{CONTRIBUIÇÕES INDIVIDUAIS}

Marcus Vinicius Pinto - Realizou o diagnóstico neurológico e a aplicação do torpedeamento.

Adriana Fiszman - Realizou a avaliação psiquiátrica.

Marcus Vinicius Pinto, Marco Antonio Alves Brasil, Mauricio de Assis Tostes e Adriana Fiszman - Contribuíram na concepção do relato do caso, na elaboração do artigo, na revisão do seu conteúdo e aprovaram sua versão final.

\section{CONFLITOS DE INTERESSE}

Não há conflitos de interesse a serem declarados.

\section{REFERÊNCIAS}

1. American Psychiatric Association. Diagnostic and Statistical Manual of Mental Disorders Fourth Edition (DSM-IV). Washington, DC: American Psychiatric Association; 1994. 
2. Kuyk J, Siffels MC, Bakvis P, Swinkels WA. Psychological treatment of patients with psychogenic non-epileptic seizures: an outcome study. Seizure. 2008;17(7):595-603.

3. Reich SG. Psychogenic movement disorders. Semin Neurol. 2006;26(3):289-96.

4. Stone J, Carson A, Sharpe M. Functional symptoms and signs in neurology: assessment and diagnosis. J Neurol Neurosurg Psychiatry. 2005;76 Suppl 1:12-12.

5. Beatty S. Non-organic visual loss. Postgrad Med J. 1999;75:201-7.

6. Tatu L, Bogousslavsky J, Moulin T, Chopard JL. The "torpillage" neurologists of World War l: electric therapy to send hysterics back to the front. Neurology. 2010;75(3):279-83.

7. Williams DT, Ford B, Fahn S. Phenomenology and psychopathology related to psychogenic movement disorders. Adv Neurol. 1995;65:231-57.

8. Poole NA, Wuerz A, Agrawal N. Abreaction for conversion disorder: systematic review with meta-analysis. Br J Psychiatry. 2010;197(2):91-5

9. Shamy MC. The treatment of psychogenic movement disorders with suggestion is ethically justified. Mov Disord. 2010;25(3):260-4.

10. Feinstein A, Stergiopoulos V, Fine J, Lang AE. Psychiatric outcome in patients with a psychogenic movement disorder: a prospective study. Neuropsychiatry Neuropsychol Behav Neurol. 2001;14(3):169-76.
11. Fiszman A, Kanner A. Comorbidities in psychogenic nonepileptic seizures: depressive, anxiety, and personality disorders. In: Schacter S, La France Jr C, editors. Gates and Rowan's Nonepileptic Seizures, 3rd. edition. Cambridge: Cambridge University Press; 2009.

12. Fiszman Alves-Leon SV, Nunes RG, D'Andrea I, Figueira I. Traumatic events and posttraumatic stress disorder in patients with psychogenic nonepileptic seizures: a critical review. Epilepsy Behav. 2004;5(6):818-25.

13. Marchetti RL, Kurcgant D, Neto JG, von Bismark MA, Marchetti LB, Fiore LA. Psychiatric diagnoses of patients with psychogenic non-epileptic seizures. Seizure. 2008;17(3):247-53.

14. Breuer J, Freud S. Edição standard brasileira das obras completas de Sigmund Freud, volume II (1893-1895): Estudos sobre a histeria, segunda edição. Rio de Janeiro: Imago Editora Ltda., 1987.

15. Freud S. Edição standard brasileira das obras completas de Sigmund Freud, volume XIV (1914-1915): A história do movimento psicanalítico, artigos sobre metapsicologia e outros trabalhos. Rio de Janeiro: Imago Editora Ltda., 1974. 